\title{
Through-thickness grain structure of metal-oxide films using EBSD
}

\author{
D.M. Feldmann*
}

* M.S. K763, Los Alamos National Laboratory, Los Alamos, NM 87544

High-temperature superconducting wires, also known as coated conductors, consist of a long flexible metal tape on top of which are deposited multiple metal-oxide buffer layers followed by a superconductor such as $\mathrm{YBa}_{2} \mathrm{Cu}_{3} \mathrm{O}_{7}$ (YBCO). In coated conductors based on a deformation textured metal tape, a.k.a. rolling assisted biaxially textured substrates (RABiTS), all metal-oxide buffer layers and the superconductor are deposited heteroepitaxially. A very high degree of texture is required in the superconductor, as the critical current density across grain boundaries decreases exponentially with increasing misorientation angle beyond an angle of $\sim 2^{\circ}[1]$.

Understanding the texture development through the oxide buffer stack and YBCO layer is key to engineering improved performance in coated conductors. This texture development has been studied by serially ion milling $\left(\mathrm{Ar}^{+}\right)$and performing automated electron backscatter diffraction scans in the same spatial location, revealing the grain structure through thickness. Spatial registration of the scans (through-thickness) was aided by laser cuts that penetrated through all metal-oxide layers.

In all cases the metal-oxide buffers were deposited by physical vapor deposition (PVD) and exhibited excellent epitaxy relative to the metal template, with the caveat of an improved out-ofplane alignment [2]. When the YBCO layer was also deposited by PVD, it replicated the buffer orientation almost perfectly (See Fig. 1). However, when the YBCO layer was deposited by an ex situ method, where a precursor was first deposited by chemical solution deposition and subsequently converted to YBCO, significant deviations in the texture of the YBCO relative to the oxide buffer template were found (See Fig. 2). Many YBCO grains overgrew the template grain boundaries, YBCO grain boundaries meandered away from template grain boundaries (up to $15 \mu \mathrm{m}$ in YBCO films only $1 \mu \mathrm{m}$ thick), and multiple YBCO grains with different orientations were found to nucleate above single template grains. Surprisingly, this deviation from epitaxial growth results in significantly improved electromagnetic properties of the superconductor, including a greatly enhanced critical current density of the coated conductor relative to YBCO deposited by PVD.

The improved electromagnetic performance of ex situ processed YBCO is due largely to the highly meandered grain boundaries that result. The grain boundaries meander along the template grain boundaries and through the thickness of the YBCO layer, creating a significantly increased interfacial area between grains, and permitted enhanced pinning of grain boundary vortex segments by grain vortex segments [3].

References

[1] D. Dimos et al., Physical Review Letters, 61 (1988) 219-222.

[2] J.D, Budai et al., Nature Materials, 2 (2003) 487-492.

[3] D.M. Feldmann et al., Journal of Applied Physics, 102 (2007) 083912.

[4] This research was supported by the Air Force Office of Scientific Research and the Department of Energy. 


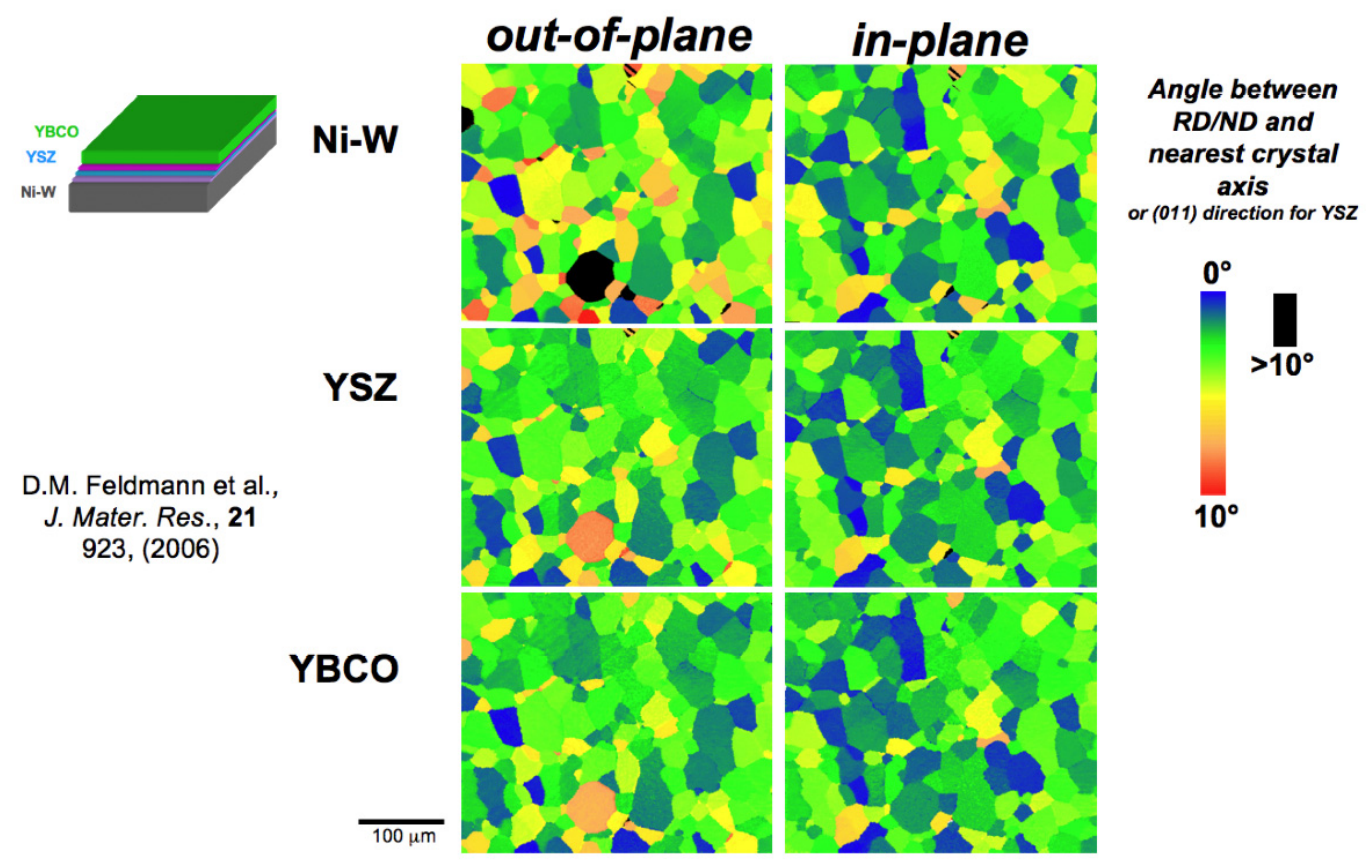

FIG. 1. Through-thickness EBSD scans of a coated conductor employing RABiTS. The YBCO was deposited by pulsed laser deposition. The in-plane texture is replicated almost perfectly throughstack. There is an out-of-plane improvement of the YSZ relative to the Ni-W metal tape.

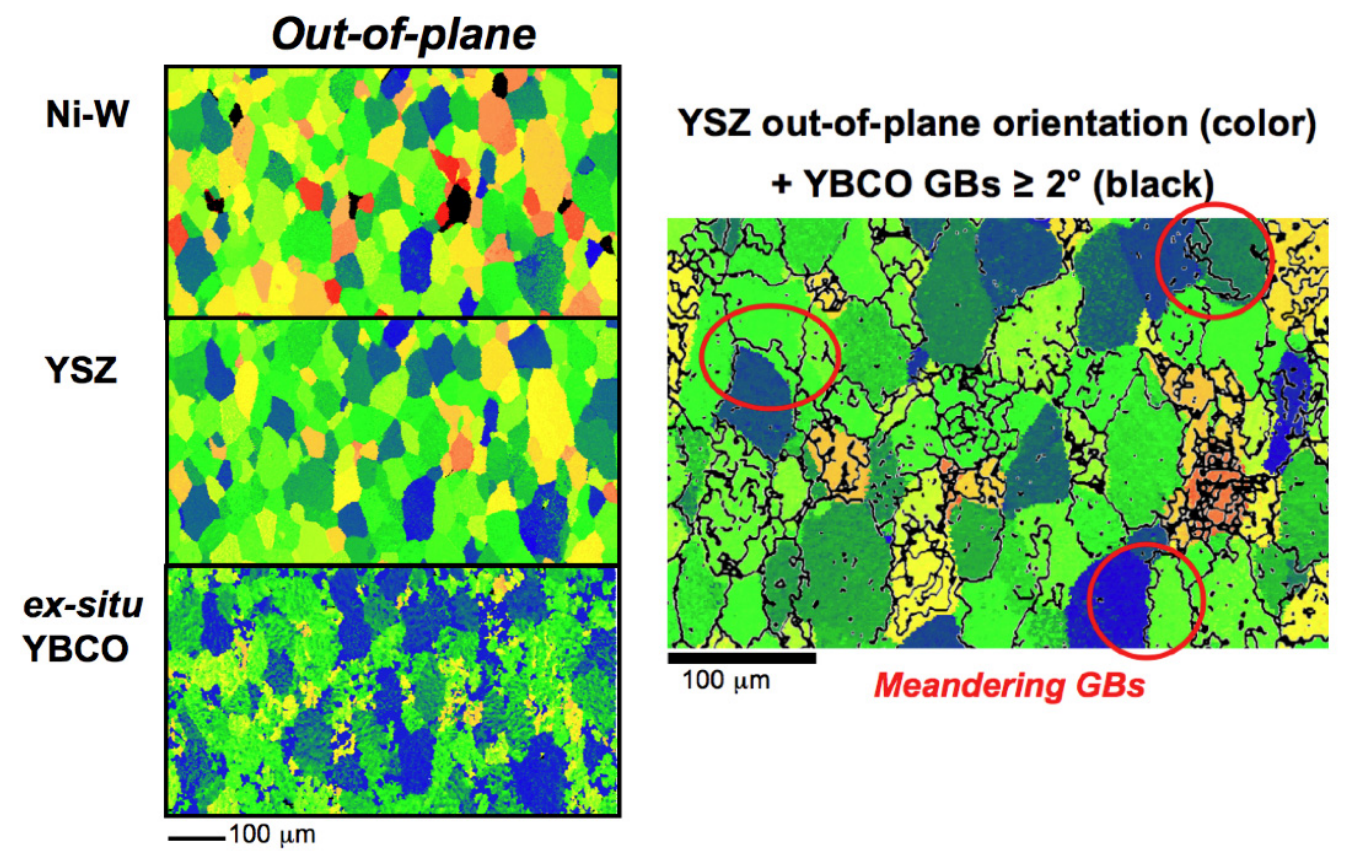

FIG. 2. Through-thickness EBSD scans of a coated conductor employing RABiTS, but this time the YBCO is deposited by an ex situ method. The legend of Fig. 1 applies. The YBCO exhibits significant deviations from the epitaxial growth (left), and many YBCO grain boundaries meander along the template grain boundaries (right). 